\title{
Pacific
}

Journal of

Mathematics

\section{SCHWARZIAN NORMS AND TWO-POINT DISTORTION}

Martin Chuaqui, Peter Duren, William Ma, Diego Mejía, David Minda AND BRad Osgood 


\title{
SCHWARZIAN NORMS AND TWO-POINT DISTORTION
}

\author{
Martin Chuaqui, Peter Duren, William Ma, \\ Diego Mejía, David Minda ANd BRAd Osgood
}

\begin{abstract}
An analytic function $f$ with Schwarzian norm $\|\mathscr{Y} f\| \leq 2\left(1+\delta^{2}\right)$ is shown to satisfy a pair of two-point distortion conditions, one giving a lower bound and the other an upper bound for the deviation. Conversely, each of these conditions is found to imply that $\|\mathscr{Y}\| \leq \mathbf{2}\left(1+\delta^{2}\right)$. Analogues of the lower bound are also developed for curves in $\mathbb{R}^{n}$ and for canonical lifts of harmonic mappings to minimal surfaces.
\end{abstract}

\section{Introduction}

A well known theorem of Nehari [16] states that if the Schwarzian derivative $\mathscr{P} f=\left(f^{\prime \prime} / f^{\prime}\right)^{\prime}-\frac{1}{2}\left(f^{\prime \prime} / f^{\prime}\right)^{2}$ of an analytic locally univalent function $f$ satisfies the inequality

$$
|\mathscr{S} f(z)| \leq \frac{2}{\left(1-|z|^{2}\right)^{2}}
$$

for all points $z$ in the unit disk $\mathbb{D}$, then $f$ is univalent in $\mathbb{D}$. The result is the best possible, since for any $\delta>0$ the weaker condition

$$
|\mathscr{\varphi} f(z)| \leq \frac{2\left(1+\delta^{2}\right)}{\left(1-|z|^{2}\right)^{2}}, \quad z \in \mathbb{D},
$$

admits functions $f$ with infinite valence. However, such functions are uniformly locally univalent in the sense that any two distinct points where $f$ assumes equal values are uniformly separated in the hyperbolic metric

$$
d(\alpha, \beta)=\frac{1}{2} \log \frac{1+\rho(\alpha, \beta)}{1-\rho(\alpha, \beta)}, \quad \text { where } \rho(\alpha, \beta)=\left|\frac{\alpha-\beta}{1-\bar{\alpha} \beta}\right| .
$$

Chuaqui, Duren, and Osgood are supported by Fondecyt grant number 1071019. Ma, Mejía, and Minda were participants in the Taft Research Seminar, 21st Century Geometric Analysis at the University of Cincinnati; Ma and Minda gratefully acknowledge partial support from the Taft Research Center.

MSC2010: primary 30C55; secondary 31A05, 53A10.

Keywords: univalent function, Schwarzian derivative, Schwarzian norm, hyperbolic metric, two-point distortion, harmonic mapping, minimal surface. 
More precisely, if $f$ satisfies the inequality (2) for some constant $\delta>0$, then $d(\alpha, \beta) \geq \pi / \delta$ for any pair of points $\alpha$ and $\beta$ in $\mathbb{D}$ where $f(\alpha)=f(\beta)$ but $\alpha \neq \beta$. Moreover, the separation constant $\pi / \delta$ is best possible. This result is essentially due to B. Schwarz [17]. A proof and further discussion can be found in [6]. Generalizations to Nehari functions other than $p(x)=\left(1-x^{2}\right)^{-2}$ are given in [6] and [8].

The Schwarzian norm of an analytic locally univalent function $f$ is defined by

$$
\|\mathscr{S} f\|=\sup _{z \in \mathbb{D}}\left(1-|z|^{2}\right)^{2}|\mathscr{S} f(z)| .
$$

Thus Nehari's theorem says that $f$ is univalent if $\|\mathscr{S} f\| \leq 2$, whereas the theorem of Schwarz says it is uniformly locally univalent if $\|\mathscr{S} f\| \leq 2\left(1+\delta^{2}\right)$ for some constant $\delta>0$.

Chuaqui and Pommerenke [4] gave a quantitative version of Nehari's theorem by showing that the condition $\|\mathscr{S} f\| \leq 2$ implies that $f$ has the two-point distortion property

$$
\Delta_{f}(\alpha, \beta)=\frac{|f(\alpha)-f(\beta)|}{\left\{\left(1-|\alpha|^{2}\right)\left|f^{\prime}(\alpha)\right|\right\}^{1 / 2}\left\{\left(1-|\beta|^{2}\right)\left|f^{\prime}(\beta)\right|\right\}^{1 / 2}} \geq d(\alpha, \beta)
$$

for all points $\alpha, \beta \in \mathbb{D}$. Conversely, they found that if $f$ satisfies (3), then $\|\mathscr{Y} f\| \leq 2$. Thus the distortion property (3) actually characterizes functions in the Nehari class.

In the present paper we show more generally that for any $\delta>0$ the analytic functions with Schwarzian norm $\|\mathscr{S} f\| \leq 2\left(1+\delta^{2}\right)$ are characterized by the local distortion property

$$
\Delta_{f}(\alpha, \beta) \geq \frac{1}{\delta} \sin (\delta d(\alpha, \beta)), \quad \alpha, \beta \in \mathbb{D}, d(\alpha, \beta) \leq \frac{\pi}{\delta} .
$$

The lower bound equals zero, as it must, when $d(\alpha, \beta)=0$ or $\pi / \delta$. Also, as $\delta \rightarrow 0$, the inequality (4) reduces to (3).

We also show that for any constant $\delta>0$ an analytic function $f$ has Schwarzian norm $\|\mathscr{S} f\| \leq 2\left(1+\delta^{2}\right)$ if and only if

$$
\Delta_{f}(\alpha, \beta) \leq \frac{1}{\sqrt{2+\delta^{2}}} \sinh \left(\sqrt{2+\delta^{2}} d(\alpha, \beta)\right), \quad \alpha, \beta \in \mathbb{D} .
$$

As a corollary, we can draw the rather surprising conclusion that for any constant $\delta>0$ and any analytic function $f$, the upper bound (5) holds for all points $\alpha, \beta \in \mathbb{D}$ if and only if the lower bound (4) holds for all $\alpha, \beta \in \mathbb{D}$ with $d(\alpha, \beta) \leq \pi / \delta$. Also, an analytic function $f$ satisfies $\Delta_{f}(\alpha, \beta) \leq(1 / \sqrt{2}) \sinh (\sqrt{2} d(\alpha, \beta))$ for all $\alpha, \beta \in \mathbb{D}$ if and only if $f$ is univalent and $\|\mathscr{Y} f\| \leq 2$.

The final section of the paper develops a generalization of the lower bound (4) for canonical lifts of harmonic mappings to minimal surfaces. 


\section{A basic lemma}

The proofs make essential use of a comparison lemma for solutions of differential equations.

Comparison Lemma. Let $Q(x)$ be continuous and $Q(x)>0$ for $x \in[0,1)$. Let $v(x)$ and $w(x)$ be defined as the solutions of

$$
\begin{aligned}
v^{\prime \prime}(x)+Q(x) v(x) & =0, \quad v(0)=0, \quad v^{\prime}(0)=1, \\
w^{\prime \prime}(x)-Q(x) w(x) & =0, \quad w(0)=0, w^{\prime}(0)=1,
\end{aligned}
$$

respectively. Suppose that $v(x)>0$ in an interval $(0, \xi)$, where $0<\xi \leq 1$. Let $p(z)$ be analytic and satisfy $|p(z)| \leq Q(|z|)$ for all $z \in \mathbb{D}$. Then the solution of

$$
u^{\prime \prime}(z)+p(z) u(z)=0, \quad u(0)=0, u^{\prime}(0)=1
$$

satisfies the inequalities

$$
v(|z|) \leq|u(z)| \quad \text { for }|z|<\xi, \quad|u(z)| \leq w(|z|) \quad \text { for all } z \in \mathbb{D} .
$$

It is clear that $w(x)>0$ for all $x \in(0,1)$, since the differential equation implies that $w^{\prime \prime}(x) \geq 0$. On the other hand, $v^{\prime \prime}(x) \leq 0$ and so it is possible that $v(x)=0$ for some $x \in(0,1)$.

The upper inequality $|u(z)| \leq w(|z|)$ was proved and applied by Essén and Keogh [12]. Herold [13] had previously obtained a more general result for differential equations of higher order. The lower inequality is essentially contained in [3], and a proof is sketched in [4]. For completeness we include detailed proofs of both inequalities here.

Proof of the Comparison Lemma. After rotation, the problem reduces to proving the inequalities for points $z$ in the real interval $0 \leq z<1$. (Let $U(r)=u\left(r e^{i \theta}\right)$ for fixed $\theta$.) To prove the upper inequality $|u(x)| \leq w(x)$ for $0 \leq x<1$, we convert the differential equation and initial conditions to an integral equation. Integration gives

$$
\begin{aligned}
u^{\prime}(x) & =1-\int_{0}^{x} p(t) u(t) d t, \\
u(x) & =x-\int_{0}^{x} \int_{0}^{y} p(t) u(t) d t d y .
\end{aligned}
$$

Reversing the order of integration, we have

so that

$$
u(x)=x-\int_{0}^{x}(x-t) p(t) u(t) d t
$$

$$
|u(x)| \leq x+\int_{0}^{x}(x-t) Q(t)|u(t)| d t, \quad 0 \leq x<1 .
$$


A similar analysis gives

$$
w(x)=x+\int_{0}^{x}(x-t) Q(t) w(t) d t, \quad 0 \leq x<1 .
$$

Subtraction now shows that $h(x)=|u(x)|-w(x)$ satisfies

$$
h(x) \leq \int_{0}^{x}(x-t) Q(t) h(t) d t, \quad 0 \leq x<1 .
$$

To infer that $h(x) \leq 0$, fix an arbitrary point $x_{0} \in(0,1)$ and let

$$
s_{0}=\sup \{s \in[0,1): h(x) \leq 0 \text { for all } x \in[0, s]\} .
$$

If $s_{0}<x_{0}$, let $M$ be the maximum value of $Q(x)$ for $0 \leq x \leq x_{0}$ and choose $x_{1} \in\left(s_{0}, x_{0}\right)$ such that $M\left(x_{1}-s_{0}\right)<1$. Let $\mu$ be the maximum value of $h(x)$ for $s_{0} \leq x \leq x_{1}$, so that $\mu=h\left(x_{2}\right)>0$ for some $x_{2} \in\left(s_{0}, x_{1}\right]$. Then

$$
\begin{aligned}
\mu=h\left(x_{2}\right) \leq \int_{0}^{x_{2}}\left(x_{2}-t\right) Q(t) h(t) d t & \leq \int_{s_{0}}^{x_{2}}\left(x_{2}-t\right) Q(t) h(t) d t \\
& \leq \int_{s_{0}}^{x_{2}}\left(x_{2}-t\right) Q(t) \mu d t \leq M\left(x_{1}-s_{0}\right) \mu<\mu,
\end{aligned}
$$

a contradiction. This shows that $s_{0} \geq x_{0}$, which proves $h(x) \leq 0$ or $|u(x)| \leq w(x)$ in $\left[0, x_{0}\right)$, hence in $[0,1)$, since the point $x_{0}$ was chosen arbitrarily in $(0,1)$. Thus $|u(z)| \leq w(|z|)$ for all $z \in \mathbb{D}$.

Now consider the lower bound $v(|z|) \leq|u(z)|$ for $|z|<\xi$. Again it suffices to carry out the proof for $z \in[0,1)$. Let $\varphi(x)=|u(x)|$, so that $\varphi^{2}=u \bar{u}$, and calculate

$$
\varphi(x) \varphi^{\prime}(x)=\frac{1}{2}\left(u^{\prime}(x) \overline{u(x)}+u(x) \overline{u^{\prime}(x)}\right)=\operatorname{Re}\left\{u^{\prime}(x) \overline{u(x)}\right\} .
$$

Hence $\left|\varphi^{\prime}(x)\right| \leq\left|u^{\prime}(x)\right|$ wherever $u(x) \neq 0$. Another differentiation gives

$$
\varphi(x) \varphi^{\prime \prime}(x)+\varphi^{\prime}(x)^{2}=\operatorname{Re}\left\{u^{\prime \prime}(x) \overline{u(x)}\right\}+\left|u^{\prime}(x)\right|^{2},
$$

from which we infer that

$$
\varphi(x) \varphi^{\prime \prime}(x) \geq \operatorname{Re}\left\{u^{\prime \prime}(x) \overline{u(x)}\right\}=-\operatorname{Re}\{p(x)\} \varphi(x)^{2},
$$

in view of the differential equation for $u$. Consequently, since $\varphi(x)=|u(x)| \geq 0$ and $|p(x)| \leq Q(x)$, we arrive at the differential inequality

$$
\varphi^{\prime \prime}(x)+Q(x) \varphi(x) \geq 0, \quad 0 \leq x<1 .
$$

On the other hand, the function $v$ satisfies the differential equation

$$
v^{\prime \prime}(x)+Q(x) v(x)=0, \quad 0 \leq x<1 .
$$


Since $v(0)=\varphi(0)=0$ and $v^{\prime}(0)=\varphi^{\prime}(0)>0$, it now follows from the Sturm comparison theorem that $\varphi(x) \geq v(x)$ up to the first zero of $v$. Thus $|u(x)| \geq v(x)$ for $0 \leq x<\xi$, and so $|u(z)| \geq v(|z|)$ for $|z|<\xi$.

\section{Distortion of analytic functions}

We turn now to the main result of this paper. It will be convenient to employ the notation $\Delta_{f}(\alpha, \beta)$ defined by (3), where $f$ is analytic and locally univalent in the disk and $\alpha, \beta \in \mathbb{D}$. It is important that this quantity is invariant under both precomposition and postcomposition with Möbius transformations. Specifically, if $\sigma$ is any Möbius automorphism of the disk, then

$$
\Delta_{f \circ \sigma}(\alpha, \beta)=\Delta_{f}(\sigma(\alpha), \sigma(\beta)), \quad \alpha, \beta \in \mathbb{D},
$$

as can be seen by direct calculation using the identity

$$
\frac{\left|\sigma^{\prime}(z)\right|}{1-|\sigma(z)|^{2}}=\frac{1}{1-|z|^{2}}, \quad z \in \mathbb{D}
$$

To show that

$$
\Delta_{T \circ f}(\alpha, \beta)=\Delta_{f}(\alpha, \beta)
$$

for every Möbius transformation $T$, it suffices to verify by simple calculation that $\Delta_{1 / f}(\alpha, \beta)=\Delta_{f}(\alpha, \beta)$, since the relation clearly holds for every affine mapping $T$.

Theorem 1. Let $f$ be analytic and locally univalent in $\mathbb{D}$ and suppose that the bound $\|\mathscr{S} f\| \leq 2\left(1+\delta^{2}\right)$ holds for some $\delta>0$. Then

$$
\Delta_{f}(\alpha, \beta) \geq \frac{1}{\delta} \sin (\delta d(\alpha, \beta))
$$

for all $\alpha, \beta \in \mathbb{D}$ with hyperbolic separation $d(\alpha, \beta) \leq \pi / \delta$, and

$$
\Delta_{f}(\alpha, \beta) \leq \frac{1}{\sqrt{2+\delta^{2}}} \sinh \left(\sqrt{2+\delta^{2}} d(\alpha, \beta)\right)
$$

for all $\alpha, \beta \in \mathbb{D}$. Each of the inequalities (7) and (8) is sharp; for each pair of points $\alpha$ and $\beta$ in the specified range, equality occurs for some function $f$ with $\|\mathscr{f}\| \leq 2\left(1+\delta^{2}\right)$. Equality holds in (7) precisely for $f=T \circ F \circ \sigma$ and in (8) for $f=T \circ G \circ \sigma$, where $F$ and $G$ are defined by

$$
F(z)=\left(\frac{1+z}{1-z}\right)^{i \delta} \text { and } G(z)=\left(\frac{1+z}{1-z}\right)^{\sqrt{2+\delta^{2}}},
$$

$\sigma$ is the Möbius automorphism of $\mathbb{D}$ with $\sigma(\alpha)=0$ and $\sigma(\beta)>0$, and $T$ is an arbitrary Möbius transformation. For each such function $f$, equality holds along the entire (admissible portion of the) hyperbolic geodesic through $\alpha$ and $\beta$. Conversely, if either inequality holds for all points $\alpha$ and $\beta$ in the specified range, then $\|\mathscr{Y} f\| \leq 2\left(1+\delta^{2}\right)$. 
Proof. The strategy is to establish the inequalities first in the special case where $\alpha=0$, then to derive them in the general case by Möbius invariance. Suppose that

$$
|\mathscr{\varphi} f(z)| \leq \frac{2\left(1+\delta^{2}\right)}{\left(1-|z|^{2}\right)^{2}}, \quad z \in \mathbb{D},
$$

for some $\delta>0$, and assume without loss of generality that $f(0)=0$ and $f^{\prime}(0)=1$. Define

Then the function

$$
g(z)=-\frac{1}{f(z)}, \quad \text { so that } \quad g^{\prime}(z)=\frac{f^{\prime}(z)}{f(z)^{2}} .
$$

$$
u(z)=\left(g^{\prime}(z)\right)^{-1 / 2}=z+c_{2} z^{2}+\cdots
$$

is analytic in $\mathbb{D}$, with $u(0)=0$ and $u^{\prime}(0)=1$, and it satisfies the differential equation

$$
u^{\prime \prime}+\left(\frac{1}{2} \mathscr{Y} f\right) u=0,
$$

since $\mathscr{Y} g=\mathscr{Y} f$. Define the functions $v(x)$ and $w(x)$ by

$$
\begin{gathered}
v^{\prime \prime}(x)+\frac{1+\delta^{2}}{\left(1-x^{2}\right)^{2}} v(x)=0, \quad v(0)=0, v^{\prime}(0)=1, \\
w^{\prime \prime}(x)-\frac{1+\delta^{2}}{\left(1-x^{2}\right)^{2}} w(x)=0, \quad w(0)=0, w^{\prime}(0)=1 .
\end{gathered}
$$

Suppose $v(x)>0$ in the interval $(0, \xi)$, where $0<\xi \leq 1$. Then in view of the hypothesis that $\left|\frac{1}{2} \mathscr{Y} f(z)\right| \leq\left(1+\delta^{2}\right)\left(1-|z|^{2}\right)^{-2}$ in $\mathbb{D}$, by the Comparison Lemma $|u(z)| \leq w(|z|)$ for all $z \in \mathbb{D}$, and $v(|z|) \leq|u(z)|$ for all $z \in \mathbb{D}$ with $|z|<\xi$.

The solutions $v(x)$ and $w(x)$ are

$$
\begin{aligned}
& v(x)=\frac{1}{\delta} \sqrt{1-x^{2}} \sin \left(\frac{\delta}{2} \log \frac{1+x}{1-x}\right), \\
& w(x)=\frac{\sqrt{1-x^{2}}}{\sqrt{2+\delta^{2}}} \sinh \left(\frac{\sqrt{2+\delta^{2}}}{2} \log \frac{1+x}{1-x}\right) .
\end{aligned}
$$

These explicit formulas can be found with reference to Kamke [14], or by means of the substitution

$$
y(t)=\frac{v(x)}{\sqrt{1-x^{2}}}, \quad \text { where } t=\frac{1}{2} \log \frac{1+x}{1-x},
$$

which reduces the first differential equation to $y^{\prime \prime}(t)+\delta^{2} y(t)=0$. Similarly, the second equation reduces to $y^{\prime \prime}(t)-\left(2+\delta^{2}\right) y(t)=0$ through the same substitution with $w$ in place of $v$.

The first positive zero of $v(x)$ occurs at the point $\xi=\tanh (\pi / \delta)$. Since

$$
u(z)=\left(g^{\prime}(z)\right)^{-1 / 2}=f(z)\left(f^{\prime}(z)\right)^{-1 / 2},
$$


the inequality $|u(z)| \geq v(|z|)$ obtained from the Comparison Lemma reduces to

$$
\frac{|f(z)|^{2}}{\left|f^{\prime}(z)\right|} \geq \frac{1}{\delta^{2}}\left(1-|z|^{2}\right) \sin ^{2}\left(\frac{\delta}{2} \log \frac{1+|z|}{1-|z|}\right)
$$

or

$$
\Delta_{f}(0, z) \geq \frac{1}{\delta} \sin (\delta d(0, z)) \quad \text { for } d(0, z) \leq \frac{\pi}{\delta} .
$$

Now let $\alpha$ and $\beta$ be arbitrary points in the unit disk and define

$$
f_{1}(z)=\frac{f(\sigma(z))-f(\alpha)}{\left(1-|\alpha|^{2}\right) f^{\prime}(\alpha)}, \quad \text { where } \sigma(z)=\frac{z+\alpha}{1+\bar{\alpha} z} .
$$

This function has the form $f_{1}=T \circ f \circ \sigma$, where $T$ is a Möbius transformation, so

$$
\Delta_{f_{1}}(0, z)=\Delta_{f \circ \sigma}(0, z)=\Delta_{f}(\sigma(0), \sigma(z))=\Delta_{f}(\alpha, \sigma(z)) .
$$

On the other hand, $\mathscr{Y} f_{1}=\mathscr{Y}(f \circ \sigma)=((\mathscr{Y} f) \circ \sigma) \sigma^{\prime 2}$, so that

$$
\left|\mathscr{S} f_{1}(z)\right|=|\mathscr{S} f(\sigma(z))|\left|\sigma^{\prime}(z)\right|^{2} \leq \frac{2\left(1+\delta^{2}\right)\left|\sigma^{\prime}(z)\right|^{2}}{\left(1-|\sigma(z)|^{2}\right)^{2}}=\frac{2\left(1+\delta^{2}\right)}{\left(1-|z|^{2}\right)^{2}} .
$$

Since $\left\|\mathscr{Y} f_{1}\right\| \leq 2\left(1+\delta^{2}\right)$ and $f_{1}(0)=0, f_{1}^{\prime}(0)=1$, it follows from what has already been proved that

$$
\Delta_{f_{1}}(0, z) \geq \frac{1}{\delta} \sin (\delta d(0, z)), \quad d(0, z) \leq \frac{\pi}{\delta} .
$$

Therefore, if $z$ is chosen so that $\sigma(z)=\beta$, we have

$$
\left.\Delta_{f}(\alpha, \beta)=\Delta_{f_{1}}(0, z) \geq \frac{1}{\delta} \sin (\delta d(\sigma(0), \sigma(z)))\right)=\frac{1}{\delta} \sin (\delta d(\alpha, \beta))
$$

for $d(\alpha, \beta) \leq \pi / \delta$, by the invariance of the hyperbolic metric under Möbius automorphisms of $\mathbb{D}$. The proof of the lower bound (7) is now complete.

The upper bound is derived in similar fashion. The Comparison Lemma gives $|u(z)| \leq w(|z|)$ for all $z \in \mathbb{D}$, which reduces to

$$
\Delta_{f}(0, z) \leq \frac{1}{\sqrt{2+\delta^{2}}} \sinh \left(\sqrt{2+\delta^{2}} d(0, z)\right) .
$$

It then follows as before that

$$
\Delta_{f}(\alpha, \beta) \leq \frac{1}{\sqrt{2+\delta^{2}}} \sinh \left(\sqrt{2+\delta^{2}} d(\alpha, \beta)\right), \quad \alpha, \beta \in \mathbb{D},
$$

by choosing $z=\sigma^{-1}(\beta)$. This proves (8).

In order to prove the sharpness of (7), we now show that for each pair of points $\alpha, \beta \in \mathbb{D}$ with $0<d(\alpha, \beta)<\pi / \delta$, there is a function $f$ with $\|\mathscr{S} f\| \leq 2\left(1+\delta^{2}\right)$ such that $\Delta_{f}(\alpha, \beta)=(1 / \delta) \sin (\delta d(\alpha, \beta))$. By Möbius invariance, it is equivalent to show that $\Delta_{F}(0, b)=(1 / \delta) \sin (\delta d(0, b))$, where $F=f \circ \sigma^{-1}$ and $\sigma$ is the Möbius automorphism of the disk for which $\sigma(\alpha)=0$ and $\sigma(\beta)=b>0$. This will 
be the case if and only if $\mathscr{G} F(z)=2\left(1+\delta^{2}\right)\left(1-z^{2}\right)^{-2}$, which is the requirement for equality in the Comparison Lemma (see [3]). Thus the general form of the extremal function is $f=T \circ F \circ \sigma$, where $F$ is a particular function (as given by (9), for instance) with Schwarzian $\mathscr{Y} F(z)=2\left(1+\delta^{2}\right)\left(1-z^{2}\right)^{-2}, \sigma$ is the Möbius automorphism defined above, and $T$ is an arbitrary Möbius transformation. Similarly, for each pair of distinct points $\alpha, \beta \in \mathbb{D}$, equality occurs in (8) precisely for functions of the form $f=T \circ G \circ \sigma$, where $G$ is a particular function (as defined by (9), for instance) with $\mathscr{S} G(z)=-2\left(1+\delta^{2}\right)\left(1-z^{2}\right)^{-2}, \sigma$ is the Möbius automorphism with $\sigma(\alpha)=0$ and $\sigma(\beta)>0$, and $T$ is an arbitrary Möbius transformation (see [12]).

Conversely, we want to show that either of the two-point distortion conditions (7) or (8) implies the bound $\|\mathscr{Y} f\| \leq 2\left(1+\delta^{2}\right)$ on the Schwarzian norm. The proofs follow an argument given by Chuaqui and Pommerenke [4] to show that the condition (3) implies $\|\mathscr{S} f\| \leq 2$. It will suffice to carry out the details only for the condition (8), because the proof for (7) is quite similar. In view of the Möbius invariance, no information is lost if we take $\alpha=0$. Without loss of generality, we may assume that $f(0)=0$ and $f^{\prime}(0)=1$, so that

$$
f(z)=z+a_{2} z^{2}+a_{3} z^{3}+\cdots .
$$

The condition (8) then reduces to

$$
\frac{|f(z)|^{2}}{\left|f^{\prime}(z)\right|} \leq \frac{1-|z|^{2}}{2+\delta^{2}} \sinh ^{2}\left(\sqrt{2+\delta^{2}} d(0, z)\right), \quad z \in \mathbb{D} .
$$

In order to conclude from (14) that $\|\mathscr{Y} f\| \leq 2\left(1+\delta^{2}\right)$, it will suffice to show that $|\mathscr{f} f(0)| \leq 2\left(1+\delta^{2}\right)$, because of the Möbius invariance. Indeed, for the function $f_{1}$ defined by (13) we have

$$
\left(1-|z|^{2}\right)^{2}\left|\mathscr{S} f_{1}(z)\right|=\left(1-|\sigma(z)|^{2}\right)^{2}|\mathscr{S} f(\sigma(z))|,
$$

and so $\left|\mathscr{S} f_{1}(0)\right|=\left(1-|\alpha|^{2}\right)^{2}|\mathscr{Y} f(\alpha)|$. But $\mathscr{Y} f(0)=6\left(a_{3}-a_{2}^{2}\right)$, so the problem reduces to showing that $\left|a_{3}-a_{2}^{2}\right| \leq \frac{1}{3}\left(1+\delta^{2}\right)$. Straightforward calculations give

$$
\begin{aligned}
& \frac{f(z)^{2}}{f^{\prime}(z)}=z^{2}\left(1+\left(a_{2}^{2}-a_{3}\right) z^{2}+\cdots\right), \\
& \frac{1-|z|^{2}}{2+\delta^{2}} \sinh ^{2}\left(\sqrt{2+\delta^{2}} d(0, z)\right)=r^{2}\left(1+\frac{1}{3}\left(1+\delta^{2}\right) r^{2}+\cdots\right), \quad r=|z| .
\end{aligned}
$$

Therefore, the inequality (14) implies

$$
\left|1+\left(a_{2}^{2}-a_{3}\right) z^{2}+O\left(r^{3}\right)\right|^{2} \leq\left|1+\frac{1}{3}\left(1+\delta^{2}\right) r^{2}+O\left(r^{3}\right)\right|^{2},
$$

which reduces to

$$
1+2 \operatorname{Re}\left\{\left(a_{2}^{2}-a_{3}\right) z^{2}+O\left(r^{3}\right)\right\} \leq 1+\frac{2}{3}\left(1+\delta^{2}\right) r^{2}+O\left(r^{3}\right) .
$$


From this we infer that

$$
\operatorname{Re}\left\{\left(a_{2}^{2}-a_{3}\right) e^{2 i \theta}\right\} \leq \frac{1}{3}\left(1+\delta^{2}\right)
$$

by setting $z=r e^{i \theta}$ for fixed $\theta$ and letting $r \rightarrow 0$. Since the angle $\theta$ can be chosen arbitrarily, we conclude that $\left|a_{3}-a_{2}^{2}\right| \leq \frac{1}{3}\left(1+\delta^{2}\right)$, as desired.

Essentially the same calculations show that if the inequality (12) holds for all $z \in \mathbb{D}$ with $d(0, z) \leq \pi / \delta$ (or equivalently for $|z| \leq \tanh (\pi / \delta)$ ), then $|\mathscr{Y} f(0)| \leq$ $2\left(1+\delta^{2}\right)$ and so $\|\mathscr{S} f\| \leq 2\left(1+\delta^{2}\right)$.

Similar results are obtained under the hypothesis $\|\mathscr{S} f\| \leq 2\left(1-\delta^{2}\right)$ for $0<\delta<1$. Then the relevant functions $v$ and $w$ of the Comparison Lemma are obtained by replacing $\delta$ by $i \delta$ in the formulas (10) and (11). Specifically,

$$
\begin{aligned}
& v(x)=\frac{1}{\delta} \sqrt{1-x^{2}} \sinh \left(\frac{\delta}{2} \log \frac{1+x}{1-x}\right), \\
& w(x)=\frac{\sqrt{1-x^{2}}}{\sqrt{2-\delta^{2}}} \sinh \left(\frac{\sqrt{2-\delta^{2}}}{2} \log \frac{1+x}{1-x}\right) .
\end{aligned}
$$

The inequalities $v(|z|) \leq|u(z)| \leq w(|z|)$ now reduce to

$$
\frac{1}{\delta} \sinh (\delta d(0, z)) \leq \Delta_{f}(0, z) \leq \frac{1}{\sqrt{2-\delta^{2}}} \sinh \left(\sqrt{2-\delta^{2}} d(0, z)\right), \quad z \in \mathbb{D},
$$

whereupon the same argument based on Möbius invariance gives

$$
\frac{1}{\delta} \sinh (\delta d(\alpha, \beta)) \leq \Delta_{f}(\alpha, \beta) \leq \frac{1}{\sqrt{2-\delta^{2}}} \sinh \left(\sqrt{2-\delta^{2}} d(\alpha, \beta)\right)
$$

for all $\alpha, \beta \in \mathbb{D}$. Conversely, if either of the inequalities in (15) holds for some $\delta \in(0,1)$ and for all $\alpha$ and $\beta$ in $\mathbb{D}$, calculations similar to the above lead to the conclusion that $\|\mathscr{Y} f\| \leq 2\left(1-\delta^{2}\right)$.

Theorem 1 was essentially proved by Mejía [15] and was discovered independently in joint work by Chuaqui, Duren, and Osgood.

\section{Distortion of harmonic mappings}

By a similar method, the lower bound (7) can be extended to harmonic mappings, or rather to their canonical lifts to minimal surfaces. The result will generalize a theorem in [9] in the case of the extremal Nehari function $p(x)=\left(1-x^{2}\right)^{-2}$. As in [9], we begin with a distortion theorem for curves in $\mathbb{R}^{n}$.

Let $\varphi:(-1,1) \rightarrow \mathbb{R}^{n}$ be a mapping of class $C^{3}$ with $\varphi^{\prime}(x) \neq 0$. The Ahlfors Schwarzian of $\varphi$ is defined by

$$
S_{1} \varphi=\frac{\left\langle\varphi^{\prime}, \varphi^{\prime \prime \prime}\right\rangle}{\left|\varphi^{\prime}\right|^{2}}-3 \frac{\left\langle\varphi^{\prime}, \varphi^{\prime \prime}\right\rangle^{2}}{\left|\varphi^{\prime}\right|^{4}}+\frac{3}{2} \frac{\left|\varphi^{\prime \prime}\right|^{2}}{\left|\varphi^{\prime}\right|^{2}},
$$


where $\langle\cdot, \cdot\rangle$ denotes the Euclidean inner product and $|\mathbf{x}|^{2}=\langle\mathbf{x}, \mathbf{x}\rangle$ for $\mathbf{x} \in \mathbb{R}^{n}$. As Ahlfors [1] observed, $S_{1}$ is invariant under postcomposition with Möbius transformations of $\mathbb{R}^{n}$. Chuaqui and Gevirtz [2] used it to give an injectivity criterion for curves. Here is a special case of their theorem.

Theorem A. Let $\varphi:(-1,1) \mapsto \mathbb{R}^{n}$ be a curve of class $C^{3}$ with tangent vector $\varphi^{\prime}(x) \neq 0$. If $S_{1} \varphi(x) \leq 2\left(1-x^{2}\right)^{-2}$, then $\varphi$ is injective.

Chuaqui and Gevirtz also showed that the arclength $s=s(x)$ of the curve $\varphi$ has Schwarzian

$$
\mathscr{S}_{S}(x)=S_{1} \varphi(x)-\frac{1}{2}\left|\varphi^{\prime}(x)\right|^{2} \kappa(x)^{2} \leq S_{1} \varphi(x),
$$

where $\kappa=\kappa(x)$ is the curvature of $\varphi$.

Our next theorem extends Theorem A to a criterion for uniform local injectivity, in the manner of B. Schwarz's extension of Nehari's theorem. Moreover, it expresses the local injectivity in quantitative form as a two-point distortion result analogous to the lower bound (7) in Theorem 1. In terms of the curve $\varphi(x)$, we define

$$
\Delta_{\varphi}(a, b)=\frac{|\varphi(a)-\varphi(b)|}{\left\{\left(1-a^{2}\right)\left|\varphi^{\prime}(a)\right|\right\}^{1 / 2}\left\{\left(1-b^{2}\right)\left|\varphi^{\prime}(b)\right|\right\}^{1 / 2}}, \quad a, b \in(-1,1) .
$$

Theorem 2. Let $\varphi:(-1,1) \mapsto \mathbb{R}^{n}$ be a curve of class $C^{3}$ with $\varphi^{\prime}(x) \neq 0$. If

then the inequality

$$
S_{1} \varphi(x) \leq \frac{2\left(1+\delta^{2}\right)}{\left(1-x^{2}\right)^{2}} \quad \text { for some } \delta>0,
$$

$$
\Delta_{\varphi}(a, b) \geq \frac{1}{\delta} \sin (\delta d(a, b))
$$

holds for all $a, b \in(-1,1)$ with $d(a, b) \leq \pi / \delta$.

Proof. First, the quantity $\Delta_{\varphi}(a, b)$ is Möbius invariant. If $\sigma$ is any Möbius automorphism of the disk that preserves the real segment $(-1,1)$, or equivalently if $\sigma$ is a Möbius automorphism with real coefficients, then

$$
\Delta_{\varphi \circ \sigma}(a, b)=\Delta_{\varphi}(\sigma(a), \sigma(b)), \quad a, b \in(-1,1) .
$$

If $T$ is any Möbius transformation of $\mathbb{R}^{n}$, then $\Delta_{T \circ \varphi}(a, b)=\Delta_{\varphi}(a, b)$. The proofs for curves are essentially the same as for analytic functions.

As in the proof of Theorem 1, we will derive the inequality (17) first for $a=0$, then deduce the general result by Möbius invariance. Because of Möbius invariance, we may assume without loss of generality that $\varphi(0)=0$ and $\left|\varphi^{\prime}(0)\right|=1$. Consider the inverted curve

$$
\Phi(x)=\frac{\varphi(x)}{|\varphi(x)|^{2}}, \quad \text { with }\left|\Phi^{\prime}(x)\right|=\frac{\left|\varphi^{\prime}(x)\right|}{|\varphi(x)|^{2}},
$$


as a straightforward calculation of $\left|\Phi^{\prime}(x)\right|^{2}$ shows. By Möbius invariance, $S_{1} \Phi=$ $S_{1} \varphi$. Recall that if $g(x)$ is a real-valued function with $g^{\prime}(x)>0$, the function $u(x)=g^{\prime}(x)^{-1 / 2}$ satisfies the differential equation $u^{\prime \prime}+\frac{1}{2}(\mathscr{g} g) u=0$. Thus if $g(x)=s(x)$, the arclength function along the curve $\Phi(x)$, then the function

$$
u(x)=\left|\Phi^{\prime}(x)\right|^{-1 / 2}=\frac{|\varphi(x)|}{\left|\varphi^{\prime}(x)\right|^{1 / 2}}
$$

satisfies $u^{\prime \prime}+\frac{1}{2}\left(\mathscr{Y}_{S}\right) u=0$ and has initial data $u(0)=0$ and $u^{\prime}(0)=1$, since $\varphi(0)=0$ and $\left|\varphi^{\prime}(0)\right|=1$. But

$$
\mathscr{Y}_{S}(x) \leq S_{1} \Phi(x)=S_{1} \varphi(x) \leq \frac{2\left(1+\delta^{2}\right)}{\left(1-x^{2}\right)^{2}},
$$

so by the Sturm comparison theorem $u(x) \geq v(x)$ for $0 \leq x \leq \tanh (\pi / \delta)$, where $v(x)$ is the function given in (10). In terms of the hyperbolic metric, this last inequality takes the form

$$
\Delta_{\varphi}(0, x) \geq \frac{1}{\delta} \sin (\delta d(0, x)), \quad d(0, x) \leq \pi / \delta,
$$

which is the desired result (17) for $a=0$. The general inequality (17) is deduced from this special case by Möbius invariance.

With the help of Theorem 2, we can now derive a two-point distortion inequality for the canonical lift of a harmonic mapping to a minimal surface. A harmonic mapping is a complex-valued harmonic function $f(z)=u(z)+i v(z)$ for $z=x+i y$ in the unit disk $\mathbb{D}$ of the complex plane. Such a mapping has a canonical decomposition $f=h+\bar{g}$, where $h$ and $g$ are analytic in $\mathbb{D}$ and $g(0)=0$. The basic properties of harmonic mappings are described in [11].

According to the Weierstrass-Enneper formulas, a harmonic mapping $f=h+\bar{g}$ with $\left|h^{\prime}(z)\right|+\left|g^{\prime}(z)\right| \neq 0$ lifts locally to a minimal surface described by conformal parameters if and only if its dilatation $\omega=g^{\prime} / h^{\prime}$ has the form $\omega=q^{2}$ for some meromorphic function $q$. The Cartesian coordinates $(U, V, W)$ of the surface are then given by

$$
U(z)=\operatorname{Re}\{f(z)\}, \quad V(z)=\operatorname{Im}\{f(z)\}, \quad W(z)=2 \operatorname{Im}\left\{\int_{0}^{z} h^{\prime}(\zeta) q(\zeta) d \zeta\right\} .
$$

We use the notation $\tilde{f}(z)=(U(z), V(z), W(z))$ for the lifted mapping from $\mathbb{D}$ to the minimal surface. The first fundamental form of the surface is $d s^{2}=\lambda^{2}|d z|^{2}$, where the conformal metric is $\lambda=\left|h^{\prime}\right|+\left|g^{\prime}\right|$.

For a harmonic mapping $f=h+\bar{g}$ with $\lambda(z)=\left|h^{\prime}(z)\right|+\left|g^{\prime}(z)\right|>0$, whose dilatation is the square of a meromorphic function, the Schwarzian derivative is defined by the formula

$$
\mathscr{S} f=2\left(\sigma_{z z}-\sigma_{z}^{2}\right), \quad \sigma=\log \lambda .
$$


If $f$ is analytic, it is easily verified that $\mathscr{S} f$ reduces to the classical Schwarzian.

In [7], the following criterion was given for the lift of a harmonic mapping to be univalent.

Theorem B. Let $f=h+\bar{g}$ be a harmonic mapping of the unit disk, with $\lambda(z)=$ $\left|h^{\prime}(z)\right|+\left|g^{\prime}(z)\right|>0$ and dilatation $g^{\prime} / h^{\prime}=q^{2}$ for some meromorphic function $q$. Let $\tilde{f}$ denote the Weierstrass-Enneper lift of $f$ to a minimal surface with Gauss curvature $K=K(\tilde{f}(z))$ at the point $\tilde{f}(z)$. Suppose that the inequality

$$
|\mathscr{S} f(z)|+\lambda(z)^{2}|K(\tilde{f}(z))| \leq \frac{2}{\left(1-|z|^{2}\right)^{2}}
$$

holds for all $z \in \mathbb{D}$. Then $\tilde{f}$ is univalent in $\mathbb{D}$.

If $f$ is analytic, its associated minimal surface is the complex plane itself, with Gauss curvature $K=0$, and the result reduces to Nehari's theorem.

In [9], Theorem $B$ was sharpened to express the univalence in the form of a two-point distortion condition. It was shown in [6] that if the bound $2\left(1-|z|^{2}\right)^{-2}$ is weakened to $2\left(1+\delta^{2}\right)\left(1-|z|^{2}\right)^{-2}$, then $\tilde{f}$ is uniformly locally univalent, the analogue of B. Schwarz's extension of Nehari's theorem. We now express the uniform local univalence in quantitative form, thus obtaining a harmonic analogue of the lower bound (7) in Theorem 1. Let

$$
\Delta_{\tilde{f}}(\alpha, \beta)=\frac{|\tilde{f}(\alpha)-\tilde{f}(\beta)|}{\left\{\left(1-|\alpha|^{2}\right) \lambda(\alpha)\right\}^{1 / 2}\left\{\left(1-|\beta|^{2}\right) \lambda(\beta)\right\}^{1 / 2}}, \quad \alpha, \beta \in \mathbb{D},
$$

where $\lambda$ is the conformal metric of the minimal surface.

Theorem 3. Let $f=h+\bar{g}$ be a harmonic mapping of the unit disk, with $\lambda(z)=$ $\left|h^{\prime}(z)\right|+\left|g^{\prime}(z)\right|>0$ and dilatation $g^{\prime} / h^{\prime}=q^{2}$ for some meromorphic function $q$. Let $\tilde{f}$ denote the canonical lift of $f$ to a minimal surface. Suppose that

$$
|\mathscr{S} f(z)|+\lambda(z)^{2}|K(\tilde{f}(z))| \leq \frac{2\left(1+\delta^{2}\right)}{\left(1-|z|^{2}\right)^{2}}, \quad z \in \mathbb{D} .
$$

Then

$$
\Delta_{\tilde{f}}(\alpha, \beta) \geq \frac{1}{\delta} \sin (\delta d(\alpha, \beta))
$$

for all $\alpha, \beta \in \mathbb{D}$ with hyperbolic separation $d(\alpha, \beta) \leq \pi / \delta$. For each pair of points $\alpha, \beta$ with $0<d(\alpha, \beta)<\pi / \delta$, equality occurs in (19) only for harmonic mappings of the form $f=h+c \bar{h}$, with $c$ a constant of modulus $|c|<1$ and $h=T \circ F \circ \sigma$, where $F$ is defined by (9), $\sigma$ is the Möbius automorphism of $\mathbb{D}$ for which $\sigma(\alpha)=0$ and $\sigma(\beta)>0$, and $T$ is an arbitrary Möbius transformation. The corresponding minimal surface is then a plane. 
Proof. The proof will apply Theorem 2. The canonical lift $\tilde{f}$ onto a minimal surface $\Sigma$ defines a curve $\tilde{f}:(-1,1) \rightarrow \Sigma \subset \mathbb{R}^{3}$. As shown in [7], the Ahlfors Schwarzian of this curve satisfies

$$
\begin{aligned}
S_{1} \tilde{f}(x) & =\operatorname{Re}\{\mathscr{Y} f(x)\}+\frac{1}{2} \lambda(x)^{2} \kappa_{e}(\tilde{f}(x))^{2}+\frac{1}{2} \lambda(x)^{2}|K(\tilde{f}(x))| \\
& \leq \operatorname{Re}\{\mathscr{S} f(x)\}+\lambda(x)^{2}|K(\tilde{f}(x))| \\
& \leq|\mathscr{S} f(x)|+\lambda(x)^{2}|K(\tilde{f}(x))|, \quad-1<x<1,
\end{aligned}
$$

where $\kappa_{e}(\tilde{f}(x))$ denotes the normal curvature of the curve at the point $\tilde{f}(x)$. Thus the hypothesis (18) tells us that $S_{1} \tilde{f}(x) \leq 2\left(1+\delta^{2}\right)\left(1-x^{2}\right)^{-2}$, and so by Theorem 2 we have the inequality

$$
\Delta_{\tilde{f}}(a, b) \geq \frac{1}{\delta} \sin (\delta d(a, b))
$$

for all $a, b \in(-1,1)$ with $d(a, b) \leq \pi / \delta$, since $\left|\tilde{f}^{\prime}(x)\right|=\lambda(x)$.

To extend the inequality (21) to arbitrary points $\alpha, \beta \in \mathbb{D}$, we appeal again to Möbius invariance. First, the quantity $\Delta_{\tilde{f}}(\alpha, \beta)$ is invariant under precomposition with Möbius automorphisms of the disk. Indeed, if $\sigma$ is any such automorphism, the composition $F=f \circ \sigma$ is a harmonic mapping with canonical lift $\tilde{F}=\tilde{f} \circ \sigma$ and conformal metric $\Lambda(z)=\lambda(\sigma(z))\left|\sigma^{\prime}(z)\right|$. Combining this with the identity (6), we see that $\Delta_{\tilde{F}}(\alpha, \beta)=\Delta_{\tilde{f}}(\sigma(\alpha), \sigma(\beta))$. Given any pair of points $\alpha, \beta \in \mathbb{D}$, choose $\sigma$ so that $\sigma(a)=\alpha$ and $\sigma(b)=\beta$ for some $a, b \in(-1,1)$. In view of (6), the hypothesis (18) is also Möbius invariant, and so $\Delta_{\tilde{F}}(a, b) \geq(1 / \delta) \sin (\delta d(a, b))$, by what we have already proved. But $d(a, b)=d(\alpha, \beta)$ by Möbius invariance of the hyperbolic metric, whereas

$$
\Delta_{\tilde{F}}(a, b)=\Delta_{\tilde{f}}(\sigma(a), \sigma(b))=\Delta_{\tilde{f}}(\alpha, \beta) .
$$

Therefore, the inequality (19) holds for all points $\alpha, \beta \in \mathbb{D}$ with $d(\alpha, \beta) \leq \pi / \delta$.

We now turn to the case of equality in (19) for two distinct points $\alpha, \beta \in \mathbb{D}$ with $d(\alpha, \beta)<\pi / \delta$. After precomposing with an automorphism of the disk, we may assume that $\alpha=0$ and $\beta=r$ with $0<r<\pi / \delta$. More precisely, if $\sigma$ is the automorphism with $\sigma(\alpha)=0$ and $\sigma(\beta)=r>0$, we need only consider equality for functions $f_{1}=f \circ \sigma^{-1}$ at the points 0 and $r$. Let $\varphi(x)=\widetilde{f}_{1}(x)$ denote the lifted curve on the corresponding minimal surface $\Sigma$. With the notation in the proof of Theorem 2, we see that equality in (19), namely $\Delta_{\tilde{f}_{1}}(0, r)=(1 / \delta) \sin (\delta d(0, r))$, is equivalent to $u(r)=(1 / \delta) \sin (\delta d(0, r))$, which by the Sturm comparison theorem can occur only if

$$
\mathscr{S}_{S}(x)=S_{1} \varphi(x)=\frac{2\left(1+\delta^{2}\right)}{\left(1-x^{2}\right)^{2}} \quad \text { for all } x \in[0, r] .
$$


But in view of (16), the equality $\mathscr{S}_{S}(x)=S_{1} \varphi(x)$ implies that the curvature $\kappa(x)$ of the curve $\varphi$ vanishes for all $x \in[0, r]$, and so that portion of the curve is a straight line in space. On the other hand, because of (20) and the hypothesis (18), the equality $S_{1} \varphi(x)=2\left(1+\delta^{2}\right)\left(1-x^{2}\right)^{-2}$ implies that the normal curvature has the property $\kappa_{e}(\varphi(x))^{2} \equiv|K(\varphi(x))|$ on $[0, r]$, so that the corresponding portion of the curve is a line of curvature of $\Sigma$. (Here we use the fact that $\Sigma$ is a minimal surface, with zero mean curvature.) But by uniqueness in the Björling problem (see [10]), a minimal surface containing a straight line segment as a line of curvature must reduce to a plane. Therefore, as shown in [5], the harmonic mapping $f_{1}$ has the form $h_{1}+c \overline{h_{1}}$ for some locally univalent analytic function $h_{1}$ and some constant $c$ with $|c|<1$. It is then easily seen that $\mathscr{Y} f_{1}=\mathscr{S} h_{1}$. Furthermore, since the surface $\Sigma$ is a plane, it has Gauss curvature $K=0$, and so (22) combines with (20) and (18) to show that

$$
\mathscr{S} h_{1}(x)=\mathscr{S} f_{1}(x)=S_{1} \widetilde{f}_{1}(x)=\frac{2\left(1+\delta^{2}\right)}{\left(1-x^{2}\right)^{2}} \quad \text { for all } x \in[0, r] .
$$

But $\mathscr{S} h_{1}$ is an analytic function, so this implies that $\mathscr{Y} h_{1}(z)=2\left(1+\delta^{2}\right)\left(1-z^{2}\right)^{-2}$ for all $z \in \mathbb{D}$. Therefore, $h_{1}=T \circ F$, where $T$ is a Möbius transformation and $F$ is a particular function (as given by (9), for instance) with Schwarzian $\mathscr{Y} F(z)=$ $2\left(1+\delta^{2}\right)\left(1-z^{2}\right)^{-2}$. Hence $f=f_{1} \circ \sigma=h+c \bar{h}$, where $h=T \circ F \circ \sigma$, as claimed. The argument also shows, as in Theorem 1, that the same functions $f$ give equality along the entire hyperbolic geodesic through $\alpha$ and $\beta$.

\section{References}

[1] L. V. Ahlfors, "Cross-ratios and Schwarzian derivatives in $\mathbb{R}^{n}$ ", pp. 1-15 in Complex analysis, edited by J. Hersch and A. Huber, Birkhäuser, Basel, 1988. MR 90a:30055 Zbl 0675.30021

[2] M. Chuaqui and J. Gevirtz, "Simple curves in $\mathbb{R}^{n}$ and Ahlfors' Schwarzian derivative", Proc. Amer. Math. Soc. 132:1 (2004), 223-230. MR 2005f:53001 Zbl 1045.53003

[3] M. Chuaqui and B. Osgood, "Sharp distortion theorems associated with the Schwarzian derivative”, J. London Math. Soc. (2) 48:2 (1993), 289-298. MR 94g:30005 Zbl 0792.30013

[4] M. Chuaqui and C. Pommerenke, "Characteristic properties of Nehari functions", Pacific J. Math. 188:1 (1999), 83-94. MR 2000d:30011 Zbl 0931.30016

[5] M. Chuaqui, P. Duren, and B. Osgood, "The Schwarzian derivative for harmonic mappings", $J$. Anal. Math. 91 (2003), 329-351. MR 2004j:30030 Zbl 1054.31003

[6] M. Chuaqui, P. Duren, and B. Osgood, "Schwarzian derivative criteria for valence of analytic and harmonic mappings", Math. Proc. Cambridge Philos. Soc. 143:2 (2007), 473-486. MR 2008i:30018 Zbl 1134.30315

[7] M. Chuaqui, P. Duren, and B. Osgood, "Univalence criteria for lifts of harmonic mappings to minimal surfaces”, J. Geom. Anal. 17:1 (2007), 49-74. MR 2008d:31001 Zbl 1211.30011

[8] M. Chuaqui, P. Duren, and B. Osgood, "Schwarzian derivatives and uniform local univalence", Comput. Methods Funct. Theory 8:1 (2008), 21-34. MR 2009j:30040 Zbl 1156.30019 
[9] M. Chuaqui, P. Duren, and B. Osgood, "Two-point distortion theorems for harmonic mappings", Illinois J. Math. 53:4 (2009), 1061-1075. MR 2741178 Zbl 1207.30035

[10] U. Dierkes, S. Hildebrandt, A. Küster, and O. Wohlrab, Minimal surfaces, I: Boundary value problems, Grundlehren der Math. Wissenschaften 295, Springer, Berlin, 1992. MR 94c:49001a Zbl 0777.53012

[11] P. Duren, Harmonic mappings in the plane, Cambridge Tracts in Mathematics 156, Cambridge University Press, Cambridge, 2004. MR 2005d:31001 Zbl 1055.31001

[12] M. Essén and F. R. Keogh, "The Schwarzian derivative and estimates of functions analytic in the unit disc", Math. Proc. Cambridge Philos. Soc. 78:3 (1975), 501-511. MR 57 \#12837 Zbl 0313.30019

[13] H. Herold, "Ein Vergleichssatz für komplexe lineare Differentialgleichungen", Math. Z. 126:1 (1972), 91-94. MR 45 \#8929 Zbl 0226.34005

[14] E. Kamke, Differentialgleichungen: Lösungsmethoden und Lösungen, I: Gewöhnliche Differentialgleichungen, 3rd ed., Mathematik und ihre Anwendungen in Physik und Technik 18 Akademische Verlagsgesellschaft, Leipzig, 1944. MR 9,33a Zbl 0061.16604

[15] D. Mejía, "Invariancia bajo transformaciones de Möbius", Habilitation thesis, Universidad Nacional de Colombia, Sede Medellín, 2007.

[16] Z. Nehari, "The Schwarzian derivative and schlicht functions", Bull. Amer. Math. Soc. 55 (1949), 545-551. MR 10,696e Zbl 0035.05104

[17] B. Schwarz, "Complex nonoscillation theorems and criteria of univalence", Trans. Amer. Math. Soc. 80 (1955), 159-186. MR 17,370e Zbl 0067.31703 
Received September 23, 2010. Revised February 1, 2011.

MARTIN CHUAQUI

FACULTAD DE MATEMÁTICAS

P. Universidad Católica de Chile

CASILLA 306

SANTIAGO 22

CHILE

mchuaqui@mat.puc.cl

PETER DUREN

DEPARTMENT OF MATHEMATICS

UNIVERSITY OF MICHIGAN

ANN ARBOR MI 48109-1043

UNITED STATES

duren@umich.edu

WILLIAM MA

SCHOOL OF INTEGRATED STUdies

PenNS ylVANia College of TeChNology

WILLIAMSPORT PA 17701

UNITED STATES

wma@pct.edu

Diego MeJÍA

DEPARTAMENTO DE MATEMÁticas

UNIVERSIDAD NACIONAL

A.A. 3840 MEDELLÍN

Colombia

dmejia@unal.edu.co

DAVID MINDA

DEPARTMENT OF MATHEMATICAL SCIENCES

UNIVERSITY OF CINCINNATI

CINCINNATI OH 45221-0025

UNITED STATES

minda@ucmail.uc.edu

BRAD OSGOOD

DEPARTMENT OF ELECTRICAL ENGINEERING

STANFORD UNIVERSITY

STANFORD CA 94305

UNITED STATES

osgood@ee.stanford.edu 


\title{
PACIFIC JOURNAL OF MATHEMATICS
}

\author{
http://pacificmath.org \\ Founded in 1951 by \\ E. F. Beckenbach (1906-1982) and F. Wolf (1904-1989)
}

\section{EDITORS}

V. S. Varadarajan (Managing Editor)

Department of Mathematics

University of California

Los Angeles, CA 90095-1555

pacific@math.ucla.edu

Vyjayanthi Chari

Department of Mathematics

University of California

Riverside, CA 92521-0135

chari@math.ucr.edu

\section{Robert Finn}

Department of Mathematics Stanford University

Stanford, CA 94305-2125

finn@math.stanford.edu

Kefeng Liu

Department of Mathematics

University of California

Los Angeles, CA 90095-1555

liu@math.ucla.edu
Darren Long

Department of Mathematics

University of California

Santa Barbara, CA 93106-3080

long@math.ucsb.edu

Jiang-Hua Lu

Department of Mathematics

The University of Hong Kong

Pokfulam Rd., Hong Kong jhlu@maths.hku.hk

Alexander Merkurjev

Department of Mathematics

University of California

Los Angeles, CA 90095-1555

merkurev@math.ucla.edu
Sorin Popa

Department of Mathematics University of California

Los Angeles, CA 90095-1555 popa@math.ucla.edu

Jie Qing

Department of Mathematics

University of California

Santa Cruz, CA 95064

qing@cats.ucsc.edu

Jonathan Rogawski

Department of Mathematics

University of California

Los Angeles, CA 90095-1555

jonr@math.ucla.edu

\section{PRODUCTION}

pacific@math.berkeley.edu

\section{SUPPORTING INSTITUTIONS}

ACADEMIA SINICA, TAIPEI

CALIFORNIA INST. OF TECHNOLOGY INST. DE MATEMÁTICA PURA E APLICADA KEIO UNIVERSITY

MATH. SCIENCES RESEARCH INSTITUTE NEW MEXICO STATE UNIV.

OREGON STATE UNIV.

\author{
STANFORD UNIVERSITY \\ UNIV. OF BRITISH COLUMBIA \\ UNIV. OF CALIFORNIA, BERKELEY \\ UNIV. OF CALIFORNIA, DAVIS \\ UNIV. OF CALIFORNIA, LOS ANGELES \\ UNIV. OF CALIFORNIA, RIVERSIDE \\ UNIV. OF CALIFORNIA, SAN DIEGO \\ UNIV. OF CALIF., SANTA BARBARA
}

\author{
UNIV. OF CALIF., SANTA CRUZ \\ UNIV. OF MONTANA \\ UNIV. OF OREGON \\ UNIV. OF SOUTHERN CALIFORNIA \\ UNIV. OF UTAH \\ UNIV. OF WASHINGTON \\ WASHINGTON STATE UNIVERSITY
}

These supporting institutions contribute to the cost of publication of this Journal, but they are not owners or publishers and have no responsibility for its contents or policies.

See inside back cover or pacificmath.org for submission instructions.

The subscription price for 2011 is US \$420/year for the electronic version, and \$485/year for print and electronic.

Subscriptions, requests for back issues from the last three years and changes of subscribers address should be sent to Pacific Journal of Mathematics, P.O. Box 4163, Berkeley, CA 94704-0163, U.S.A. Prior back issues are obtainable from Periodicals Service Company, 11 Main Street, Germantown, NY 12526-5635. The Pacific Journal of Mathematics is indexed by Mathematical Reviews, Zentralblatt MATH, PASCAL CNRS Index, Referativnyi Zhurnal, Current Mathematical Publications and the Science Citation Index.

The Pacific Journal of Mathematics (ISSN 0030-8730) at the University of California, c/o Department of Mathematics, 969 Evans Hall, Berkeley, CA 94720-3840, is published monthly except July and August. Periodical rate postage paid at Berkeley, CA 94704, and additional mailing offices. POSTMASTER: send address changes to Pacific Journal of Mathematics, P.O. Box 4163, Berkeley, CA 94704-0163.

PJM peer review and production are managed by EditFLOW ${ }^{\mathrm{TM}}$ from Mathematical Sciences Publishers.

PUBLISHED BY PACIFIC JOURNAL OF MATHEMATICS

at the University of California, Berkeley 94720-3840

A NON-PROFIT CORPORATION

Typeset in LATEX

Copyright $(2011$ by Pacific Journal of Mathematics 


\section{PACIFIC JOURNAL OF MATHEMATICS}

Volume $254 \quad$ No. $1 \quad$ November 2011

A mean curvature estimate for cylindrically bounded submanifolds

Luis J. AlíAS and MARCos DAJCZER

Weyl group multiple Dirichlet series of type $C$

JENNIFER BEINEKE, BENJAMIN BRUBAKER and SHARON FrECHETTE

Milnor open books of links of some rational surface singularities

MOHAN BHUPAL and BURAK OZBAGCI

Simple closed curves, word length, and nilpotent quotients of free groups

KHALID BOU-RABEE and ASAF HADARI

Strong submodules of almost projective modules

GÁBOR BRAUN and JAN TRLIFAJ

Interlacing log-concavity of the Boros-Moll polynomials

William Y. C. Chen, LARry X. W. WAng and ERnest X. W. Xia

Schwarzian norms and two-point distortion

Martin Chuaqui, Peter Duren, William Ma, Diego Mejía, David

MINDA and BRAD OSGOOD

The principle of stationary phase for the Fourier transform of $D$-modules

JIANGXUE FANG

Monotonicity and uniqueness of a 3D transonic shock solution in a conic nozzle with

variable end pressure

JUN LI, ZHOUPING XIN and HUICHENG YIN

Refined open noncommutative Donaldson-Thomas invariants for small crepant resolutions

KENTARO NAGAO

The Dirichlet problem for harmonic functions on compact sets

TONY L. PERKINS

Extension of an analytic disc and domains in $\mathbb{C}^{2}$ with noncompact automorphism group

\section{MINJU SONG}

Regularity of the first eigenvalue of the $p$-Laplacian and Yamabe invariant along geometric flows

ER-MIN WANG and YU ZHENG 